\title{
ALMA CO(2-1) observations in the XUV disk of M83
}

\author{
Isadora C. Bicalho ${ }^{1}$, Francoise Combes ${ }^{1,2}$, Monica Rubio $^{3}$, Celia Verdugo ${ }^{4}$, and Philippe Salome ${ }^{1}$ \\ 1 Observatoire de Paris, LERMA, CNRS, PSL Univ., UPMC, Sorbonne Univ., 75014 Paris, France \\ e-mail: isadora.bicalho@gmail.com \\ 2 College de France, 11 Place Marcelin Berthelot, 75005 Paris, France \\ e-mail: francoise. combes@obspm.fr \\ 3 Departamento de Astronomía, Universidad de Chile, Chile \\ e-mail: mrubio@das.uchile.cl \\ 4 Joint ALMA Observatory, Santiago, Chile
}

Received 23 November 2017 / Accepted 3 July 2018

\begin{abstract}
The extended ultraviolet (XUV) disk galaxies are some of the most interesting objects studied in the last few years. The UV emission, revealed by GALEX, extends well beyond the optical disk after the drop in $\mathrm{H} \alpha$ emission, the usual tracer of star formation. This shows that sporadic star formation can occur in a large fraction of the HI disk at radii up to 3 or 4 times the optical radius. In most galaxies, these regions are poor in stars and are dominated by under-recycled gas; they therefore bear some similarity to the early stages of spiral galaxies and high-redshift galaxies. One remarkable example is M83, a nearby galaxy with an extended UV disk reaching 2 times the optical radius. It offers the opportunity to search for molecular gas and to characterize the star formation in outer disk regions, traced by the UV emission. We obtained $\mathrm{CO}(2-1)$ observations with ALMA of a small region in a $1.5^{\prime} \times 3^{\prime}$ rectangle located at $r_{\mathrm{gal}}=7.85^{\prime}$ over a bright UV region of M83. There is no CO detection, in spite of the abundance of HI gas, and the presence of young stars traced by their HII regions. Our spatial resolution $(17 \mathrm{pc} \times 13 \mathrm{pc})$ was perfectly fitted to detect giant molecular clouds (GMC), but none were detected. The corresponding upper limits occur in a region of the Kennicutt-Schmidt diagram where dense molecular clouds are expected. Stacking our data over HI-rich regions, using the observed HI velocity, we obtain a tentative detection corresponding to $\mathrm{a} \mathrm{H}_{2}$-to-HI mass ratio of $<3 \times 10^{-2}$. A possible explanation is that the expected molecular clouds are CO-dark because of the strong UV radiation field. This field preferentially dissociates $\mathrm{CO}$ with respect to $\mathrm{H}_{2}$, due to the small size of the star-forming clumps in the outer regions of galaxies.
\end{abstract}

Key words. galaxies: spiral - galaxies: ISM - galaxies: star formation - ISM: molecules - galaxies: clusters: individual: M83

\section{Introduction}

Over the last decades extended ultraviolet (XUV) disk galaxies have gained interest. Gil de Paz et al. (2005) shows the presence of UV-bright complexes in the outermost of some galaxy disks. These are called XUV disk galaxies, hosting UV emission well beyond their optical radii. UV-bright disks extending up to 3-4 times their optical radius $\left(R_{25}\right)$ have been reported in about $30 \%$ of spiral galaxies (Thilker et al. 2005a; Gil de Paz et al. 2007b). Their extended UV emission covers a significant fraction of the area detected in $\mathrm{HI}$ at $21 \mathrm{~cm}$ wavelength (Bigiel et al. 2010; Boissier et al. 2003; Zaritsky \& Christlein 2007). Generally, the UV star formation (SF) is related to this extending HI structure, for example showing evidence of metal enrichment (Gil de Paz et al. 2007a). Their far-ultraviolet (FUV) and nearultraviolet (NUV) colours are generally consistent with young populations of $\mathrm{O}$ and $\mathrm{B}$ stars, which probe a wider range of ages than $\mathrm{H}_{\alpha}$, and at low SF levels the number of ionizing stars may be very small (Dessauges-Zavadsky et al. 2014; Boissier et al. 2003).

Studying star formation beyond the optical radius allows us to address the condition of low-metallicity environments (Bigiel et al. 2010). The study of SF in nearby galaxies involves the star formation rate (SFR), its surface density $\left(\Sigma_{\mathrm{SFR}}\right)$ and the gas surface density $\left(\Sigma_{\text {gas }}\right)$ including both molecular and atomic gas. The relation between these quantities is known as the Kennicutt-Schmidt $(\mathrm{KS})$ relation $\left(\Sigma_{\mathrm{SFR}} \propto \Sigma_{\text {gas }}^{n}\right.$; Schmidt 1959; Kennicutt 1998; Kennicutt \& Evans 2012). This relation describes how efficiently galaxies turn their gas into stars in quantifying the star formation efficiency (SFE). The KS relation is almost linear when most of the gas is molecular, providing a constant gas consumption timescale of about $3 \mathrm{Gyr}$ (e.g. Bigiel et al. 2011; Saintonge et al. 2011). The SFE falls very quickly when the $\Sigma_{\text {gas }}<10 M_{\odot} \mathrm{pc}^{2}$ when the gas is mainly atomic. However, recent surveys of molecular gas at high resolution have the sensitivity to probe this relation at $\Sigma_{\text {gas }} \lesssim 3 M_{\odot} \mathrm{pc}^{2}$. Studies of SF beyond the optical radius primarily concentrated on comparing different SF tracers. Until now studies of SFE in XUV disks were principally focused on atomic gas. These environments are not propitious to $\mathrm{H}_{2}$ formation due to the low gas density and low metallicity, levels similar to galaxies in the early universe.

The confirmed occurrence of star formation in the outer disk of normal spirals has several important implications; it indicates the presence of molecular gas in the outskirts of spirals, possibly an efficient phase transition from $\mathrm{HI}$ to $\mathrm{H}_{2}$. It is one of the regions where the unresolved issue of the atomic hydrogen gas origin can be studied: either $\mathrm{HI}$ is the main phase, which can be transformed to $\mathrm{H}_{2}$ to form stars, or it is the product of the star formation process, i.e. the result of the photodissociation of $\mathrm{H}_{2}$ by the UV flux radiation emanated from newly formed stars (Allen et al. 2004; Smith et al. 2000). It provides a simplified laboratory for 
determining the star formation threshold. It is also the place where star formation can be studied in quiescent and lowmetallicity environments that may affect the SFE and the initial mass function.

Crosthwaite et al. (2002) studied the overall molecular gas morphology of M83 with $\mathrm{CO}(1-0)$ and $\mathrm{CO}(2-1)$ covering the $14^{\prime} \times 14^{\prime}$ optical disk. In this study they found that $\mathrm{CO}$ falls rapidly at a radius of $5^{\prime}=7 \mathrm{kpc}$, and they proposed that this is also the decline in the total gas surface density, even if the HI emission continues further out but with a lower surface density. Molecular gas dominates inside $7 \mathrm{kpc}$ radius ( $80 \%$ of the gas), while in total it is only $30 \%$ of the gas. The $\mathrm{CO}(2-1) / \mathrm{CO}(1-0)$ intensity ratio is $\sim 1$. At $7 \mathrm{kpc}$, where the disk begins to warp, the ISM pressure might reach a threshold for the formation of molecular clouds.

Thilker et al. (2005b) have modified this view with GALEX observations: diffuse UV emission is detected beyond the bright star-forming disk when $\mathrm{H} \alpha$ and $\mathrm{CO}$ emission drop. This discovery made M83 the prototype of XUV disk galaxies. Koda et al (2012) report deep Subaru $\mathrm{H} \alpha$ observations of the extended ultraviolet disk of M83, and found some weak emission, not seen by Thilker et al. (2005b). Dong et al. (2008) show with Spitzer that the SF has been an ongoing process in the extreme outer parts of M83 for at least $1 \mathrm{Gyr}$. In a comparison between $\mathrm{HI}$ and FUV emission, Bigiel et al. (2010) show that the most extended atomic gas observed in M83 will not be consumed by in situ SF, and this might be due to the low efficiency of the HI-to- $\mathrm{H}_{2}$ phase transition there. However, the present low SF might be sufficient for chemical enrichment. A flat oxygen abundance gradient was obtained beyond $R_{25}$ by Bresolin et al. (2009): they find only a slight decline in abundance beyond this galoctocentric distance with $12+\log (\mathrm{O} / \mathrm{H})$ between 8.2 and 8.6. Bresolin et al. (2016) present a chemical evolution model to reproduce the radial abundance gradient of M83 in $R_{25}$, and their model is able to quantify the metallicity of the gas, which is very close to that of the stars.

Star formation in low gas surface density, less than $10 M_{\odot} \mathrm{pc}^{-2}$, is not very well known. The SFE in these regions is very low and seems to be uncorrelated with $\Sigma_{\text {gas }}$; the SFR has a much larger dynamical range than the local surface density (Boissier et al. 2007). CO observations in such environments are rare, due the weakness of the emission. A robust, quantitative picture of how the environment in the outer disks affects star formation is crucial to understanding the origins of galaxy structure. Dessauges-Zavadsky et al. (2014) were the first to study the molecular SFE in an XUV disk, M63, where they detected $\mathrm{CO}(1-0)$ in 2 of 12 pointings using the IRAM $30 \mathrm{~m}$ telescope. The authors concluded that the molecular gas in those regions has a low SFE compared to regions in the inner disk. There are only four galaxies with molecular detections in the outermost disks beyond $R_{25}$ : M63 and in addition NGC 4414 (Braine \& Herpin 2004), NGC 6946 (Braine et al. 2007), M33 (Gratier et al. 2010). NGC 4625 has been actively searched for CO emission, but it has not been detected (Watson et al. 2016). In these papers, the SFE is defined as $\Sigma_{\mathrm{SFR}} / \Sigma_{\mathrm{H}_{2}}$, and we adopt this definition here (Dessauges-Zavadsky et al. 2014).

In this paper, we present ALMA $\mathrm{CO}(2-1)$ data covering one region outside the optical disk of M83. We describe our ALMA observations in greater detail in Sect. 2, and our results in Sect. 3. Section 4 presents the discussion of the SFR in low-density and low-metallicity environments and physical reasons to explain the lack of $\mathrm{CO}$ emission in the outer M83 disk.
Table 1. M83 observations.

\begin{tabular}{lc}
\hline \hline & Values \\
\hline RA (centre) & $13^{\mathrm{h}} 37^{\mathrm{m}} 03.6^{\mathrm{s}}$ \\
Dec (centre) & $-29^{\mathrm{d}} 59^{\prime} 47.6^{\prime \prime}$ \\
Distance (Mpc) & 4.8 \\
$R_{\text {observed }}$ & $7.85^{\prime}=11 \mathrm{kpc}$ \\
Synthesized beam & $0.75^{\prime \prime} \times 0.56^{\prime \prime}=17 \times 13 \mathrm{pc}$ \\
\hline
\end{tabular}

\section{Observations}

We observed $\mathrm{CO}(2-1)$ emission at $229.67 \mathrm{GHz}$ (band 6) in M83 with ALMA during Cycle 2 (PI: Monica Rubio). The selected region is located at $13^{\mathrm{h}} 37^{\mathrm{m}} 03.6^{\mathrm{s}}-29^{\mathrm{d}} 59^{\prime} 47.6^{\prime \prime}$ enclosed in a $3^{\prime} \times 1.5^{\prime}(4 \mathrm{kpc} \times 2 \mathrm{kpc})$ rectangle located at $r_{\mathrm{gal}}=7.85^{\prime}=11 \mathrm{kpc}$ from M83 centre. We used the selection criteria corresponding to FUV/NUV GALEX images (Gil de Paz et al. 2007a), using the peaks of UV emission, as well as the correlation with the HI emission from the THINGS survey (Walter et al. 2008). By choosing these peaks of emission we focused on the outer parts of the UV disks, beyond the $r_{25}$ optical radius, where we are interested in detecting the molecular gas (see Fig. 1).

These selection criteria were used for the M63 XUV disk (NGC 5055), and led to a CO detection (Dessauges-Zavadsky et al. 2014) far outside the $r_{25}$ limit, while Schruba et al. (2011) only obtained an upper limit at $300^{\prime \prime}$ from the galactic centre. The selected region of M83 was observed for $\sim 1 \mathrm{~h}$ in March 2014 in very good weather conditions (pwv $1.3 \mathrm{~mm}$ ). The $12 \mathrm{~m}$ array was used with 34 antennas and a maximum baseline of $558.2 \mathrm{~m}$. The map was prepared with a mosaic of 121 pointings separated by $12.9^{\prime \prime}$ (Fig. 2), and with an integration time of $10.8 \mathrm{~s}$ per pointing.

The data were calibrated using the CASA reduction package. Approximately $36 \%$ of the data were flagged, which was current at this epoch for $12 \mathrm{~m}$ Array data. We produced a $\mathrm{CO}(2-1)$ data cube for each pointing with natural weighting, and for a velocity range from 15 to $1015 \mathrm{~km} \mathrm{~s}^{-1}$, a channel spacing of $2.5 \mathrm{~km} \mathrm{~s}^{-1}$, and an rms of $10.3 \mathrm{mJy}$ per channel. The calibrated $121 \mathrm{uv}$-tables were subsequently exported to GILDAS where the cleaning and the cube analysis were performed.

There is no continuum detection, except one weak $(<2 \mathrm{mJy})$ continuum (point) source, most probably a background source, at $\mathrm{RA}=13^{\mathrm{h}} 37^{\mathrm{m}} 00.79^{\mathrm{s}}$, Dec $=-30^{\circ} 00^{\prime} 10.8^{\prime \prime}$. Using natural weighting the synthesized beamsize of the continuum map was $0.78^{\prime \prime} \times 0.60^{\prime \prime}$, with $\mathrm{rms}=0.19 \mathrm{mJy}$.

For the $\mathrm{CO}$ line we can estimate the upper limits found in each beam, assuming a profile width of $15 \mathrm{~km} \mathrm{~s}^{-1}$ FWHM. The rms noise level is $4 \mathrm{mJy}$ in $15 \mathrm{~km} \mathrm{~s}^{-1}$ channels for each beam of $0.75^{\prime \prime} \times 0.56^{\prime \prime}$. The $3 \sigma$ upper limit of the integrated emission is therefore $0.180 \mathrm{Jy} \mathrm{km} \mathrm{s}^{-1}$. This corresponds to $\mathrm{L}^{\prime} \mathrm{CO}(2-1)=2.5 \times 10^{3} \mathrm{~K} \mathrm{~km} \mathrm{~s}^{-1} \mathrm{pc}^{2}$. Assuming an intensity ratio of $I_{21} / I_{10} 0.7$ (e.g. Braine \& Combes 1992), and a standard CO-to- $\mathrm{H}_{2}$ conversion factor $\alpha=4.36 M_{\odot} /\left(\mathrm{K} \mathrm{km} \mathrm{s}^{-1} \mathrm{pc}^{2}\right)$, this corresponds to $M\left(\mathrm{H}_{2}\right)=1.5 \times 10^{4} M_{\odot}$. This mass scale is much lower than a giant molecular cloud (GMC) in the beam of $17 \times 13 \mathrm{pc}$.

Another way to interpret this limit is to consider that our $10.3 \mathrm{mJy} \mathrm{beam}^{-1}$ noise level in $2.5 \mathrm{~km} \mathrm{~s}^{-1}$ channels corresponds to a brightness temperature of $0.55 \mathrm{~K}$. Therefore, clouds of $1.6 \mathrm{~K}$ should have been detected at $3 \sigma$. In the outer parts of the Milky Way, as far as $R=20 \mathrm{kpc}$ from the Galactic centre, molecular clouds up to $10 \mathrm{~K}$ (in $\mathrm{CO}(1-0)$ ) and $5 \mathrm{~K}$ (in $\mathrm{CO}(2-1)$ ) have been observed at similar widths 


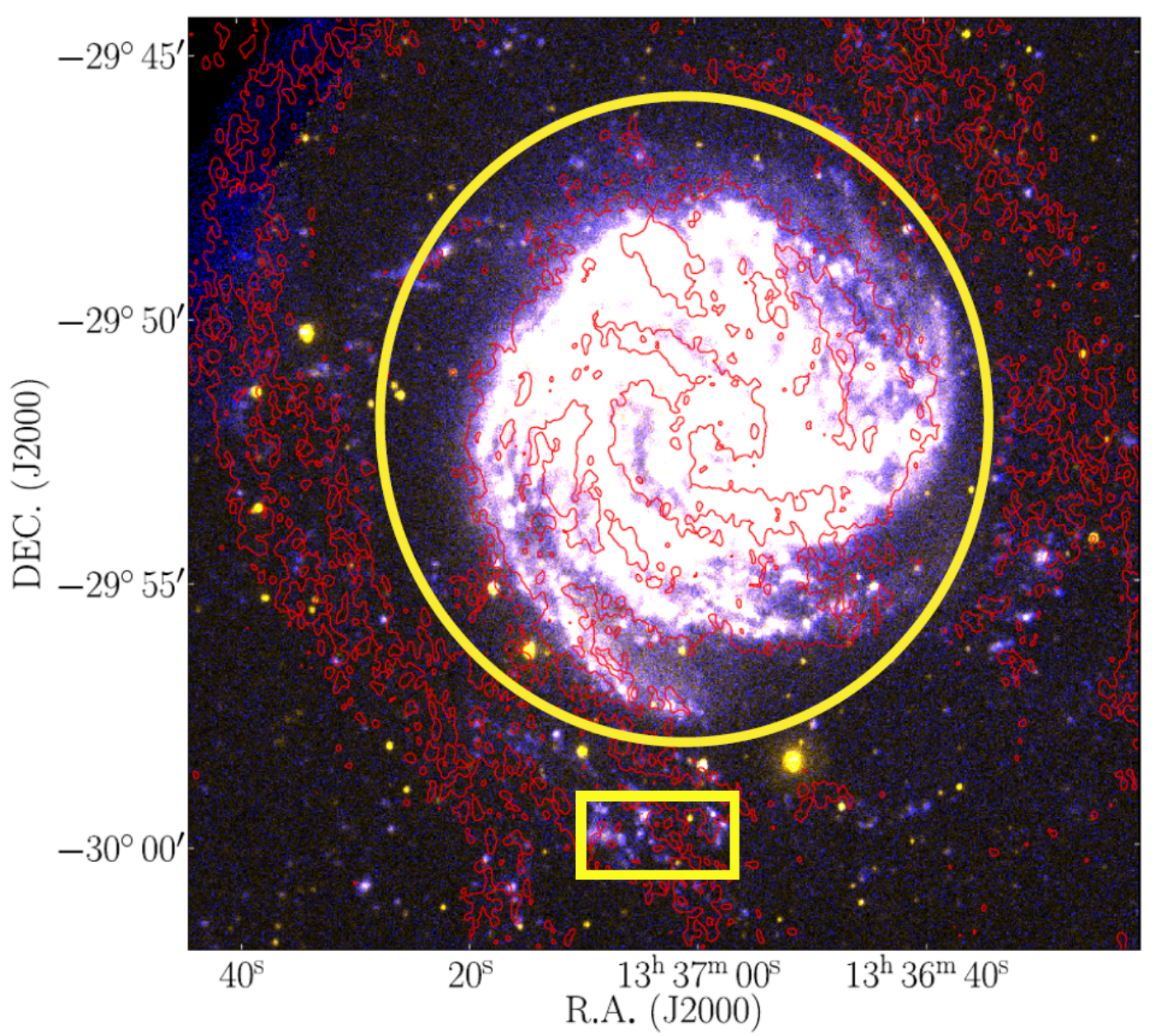

Fig. 1. GALEX FUV image (background) of M83 with HI contours (red). The HI emission is from the THINGS survey. The yellow ellipse shows the optical radius $\left(r_{25}=6.09^{\prime}=8.64 \mathrm{kpc}\right)$ and the rectangle indicates the region observed with ALMA and analysed in this paper, located at $r_{\text {gal }}=7.85^{\prime}=11 \mathrm{kpc}$.

(e.g. Digel et al. 1994; Sun et al. 2015, 2017). They would have been detected quite easily in our survey.

\section{Results}

We searched in an automatic way for CO (2-1) emission with a $\mathrm{S} / \mathrm{N}$ of more than $5 \sigma$ in each of the 121 cubes, with the "detection assessment" procedure in GILDAS. We found 14 possibilities of emission, falling in the HI range of velocities (500$700 \mathrm{~km} \mathrm{~s}^{-1}$ ) in this region of the galaxy, out of the 121 cubes. However, there is no spatial coincidence between these hints of emission and any of the other tracers. Also, the width of the profiles are in general too large. Therefore, evidence for the $\mathrm{CO}$ emission from molecular clouds is very weak.

In contrast, we look for the most prominent clumps from other tracers, in particular $\mathrm{H} \alpha$. Using the observations from the Subaru telescope (Koda et al. 2012) we found 13 regions of star formation, and tried to search for $\mathrm{CO}$ emission there. These regions are shown in Fig. 3, where the circles show the spatial regions corresponding to our choice. After looking at the ALMA data, none of the hints found have more than a $3 \sigma$ signal.

\subsection{Matched filter technique}

The reliable detection of weak signals is an issue in astronomical data. The data analysis process can lead us to underestimate the probability of false detection, which is why it is necessary to estimate it. A technique that is expected to have among the best probabilities of true detection is the matched filter (MF) technique. The goal of the filter is to maximize the detectability of the signal of a known structure inside a random noise Gaussian (Vio \& Andreani 2016). To better comprehend the quality of our observations (e.g. to find out whether our measurements are biased) we first apply a simple technique. Here we want to check the assumption that the probability density function (PDF) of the noise peaks is close to a Gaussian. In Vio \& Andreani (2016), they show from a zero-mean map that when the positions and number of sources are unknown the matched filter might underestimate the probability of false detections. We use here the simplest technique: we plotted the pixel values from our moment 0 data. The values are plotted in Fig. 4. The plot shows no obvious irregularity or departure from a Gaussian, revealing no problem with the cleaning or reduction. Since we know that the signal should correlate to the other ISM tracer, the HI- $21 \mathrm{~cm}$ emission, which gives us the spectral region to find the line, we apply this filter to the data through a stacking technique (see following section).

\subsection{Stacking of CO spectra, according to the HI velocity}

The $\mathrm{CO}$ emission is normally associated with $\mathrm{HI}$ peaks because the host molecular clouds probably form in regions of relatively 


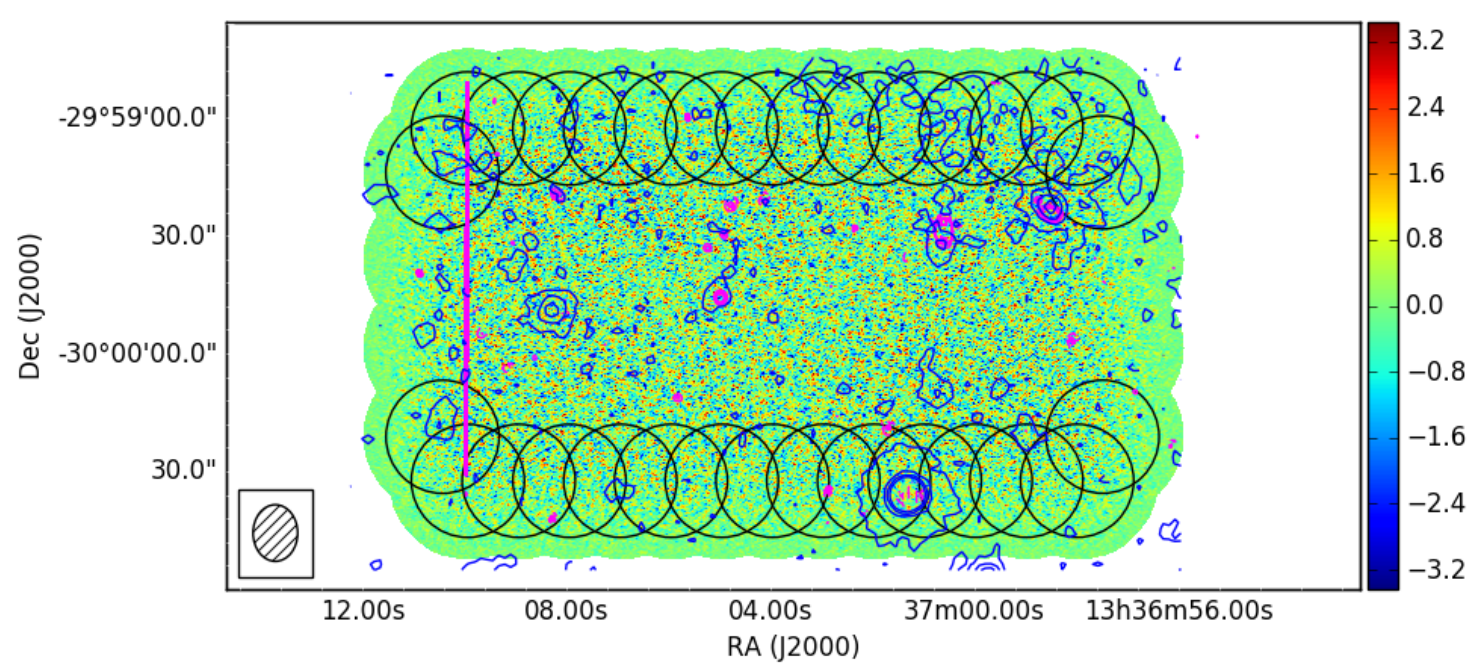

Fig. 2. Scheme of the ALMA mosaic of our observations. The black circles of 27" diameter show the positions of the 121 pointings used to map the $\mathrm{CO}(2-1)$ emission. The background image is the $\mathrm{CO}$ integrated emission (zero moment of the data cube). The contours are H $\alpha$ (magenta) and FIR $24 \mu \mathrm{m}$ (black).

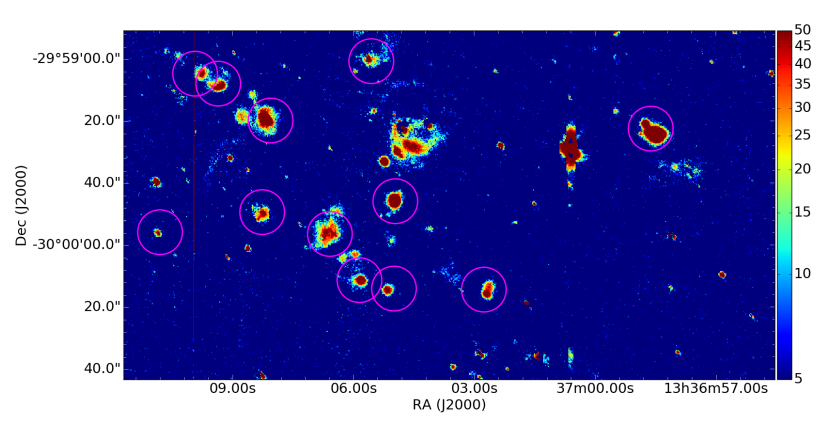

Fig. 3. $\mathrm{H}_{\alpha}$ image of the region from Subaru (Koda et al. 2012). The magenta circles show the positions where $\mathrm{CO}$ was searched.

high HI column density in the absence of spiral density waves (Gardan et al. 2007). Therefore, to exploit the full mosaic of data from ALMA, we must stack all individual spectra where emission is expected at the HI expected velocity. Averaging all pixels of the map has not given any signal; this might be due to velocity dilution since there is a significant velocity gradient of $\sim 100 \mathrm{~km} \mathrm{~s}^{-1}$ over the corresponding HI map (see Fig. 5, right). For this $\mathrm{CO}$ averaged spectrum, we find an rms of $0.017 \mathrm{mJy}$ in $14 \mathrm{~km} \mathrm{~s}^{-1}$ channels. We therefore computed the first moment of the HI cube to have an average velocity at each $1.5^{\prime \prime} \times 1.5^{\prime \prime}$ pixel of the map. We smoothed the $\mathrm{CO}(2-1)$ map at the $1.5^{\prime \prime}$ resolution, and for each beam we shifted the spectra of the right velocity amount to have all of them centred at the expected velocity known from the HI spectrum. Then we averaged all spectra where the HI integrated intensity was above a certain threshold, which was $50 \mathrm{mJy}$ beam $\times \mathrm{km} \mathrm{s}^{-1}$. The same stacking was done on the HI cube, which gives the result plotted in Fig. 5, left. The resulting HI stacked spectrum has a FWHM of $33 \mathrm{~km} \mathrm{~s}^{-1}$. No baseline was subtracted from the stacked spectra. The stacked $\mathrm{CO}(2-1)$ spectrum shows a hint of emission (at $3.5 \sigma$ ) with a FWHM of $14 \mathrm{~km} \mathrm{~s}^{-1}$. When properly reduced, the average integrated flux is $0.85 \mathrm{mJy} \mathrm{km} \mathrm{s}^{-1}$. Adopting the conversion factor described in Sect. 2, this low value corresponds to an average mass of $65 M_{\odot}$ of molecular gas per beam, but spread over $3 \times 10^{4}$ beams; therefore, it would represent a total mass of $2 \times 10^{6} M_{\odot}$ over the whole mapped region of $4 \times 2 \mathrm{kpc}$.

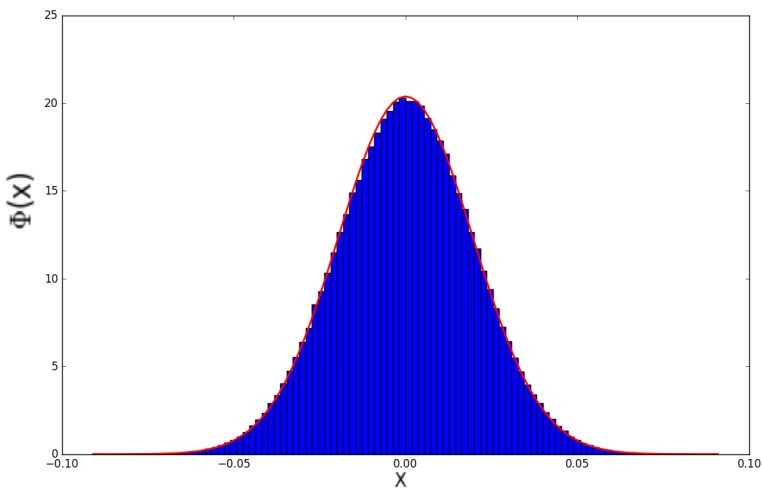

Fig. 4. Histogram of the pixel values from the ALMA data cube (our observations). The blue histogram comes from the pixel values and the red curve is a Gaussian fit. We see that the noise is a perfect Gaussian which does not show any indication of bumps that could be related to the reduction and deconvolution procedures.

The same computation can be done on the averaged stacked HI spectrum. With a flux of $0.1 \mathrm{Jy}$ beam $\mathrm{km} \mathrm{s}^{-1}$, and a beam of $15.2^{\prime \prime} \times 11.4^{\prime \prime}$, this gives a total HI mass of $7.0 \times 10^{7} M_{\odot}$ over the same region. The mass ratio of the molecular to the atomic gas is then $M\left(\mathrm{H}_{2}\right) / M(\mathrm{HI})=3 \times 10^{-2}$ or lower.

It is important to note that the stacking technique is not used here to smooth out possible extended signal on scales that would in any case be filtered out by the interferometer. On the contrary, here we aim to conserve our spatial resolution of $17 \mathrm{pc} \times 13 \mathrm{pc}$ fitted to GMC scale in order to avoid dilution of the cloud emission. The stacking, as usual, averages out several realizations of possible cloud emission.

\section{Discussion}

The extended ultraviolet disks, present in $10 \%$ of nearby galaxies, offer the opportunity to study the interstellar medium and star formation in extreme conditions with low average gas density and surprisingly abundant star formation. M83 was one of the first XUV detections in its outer regions and it is the prototype for this type of galaxy. However, no highly significant 

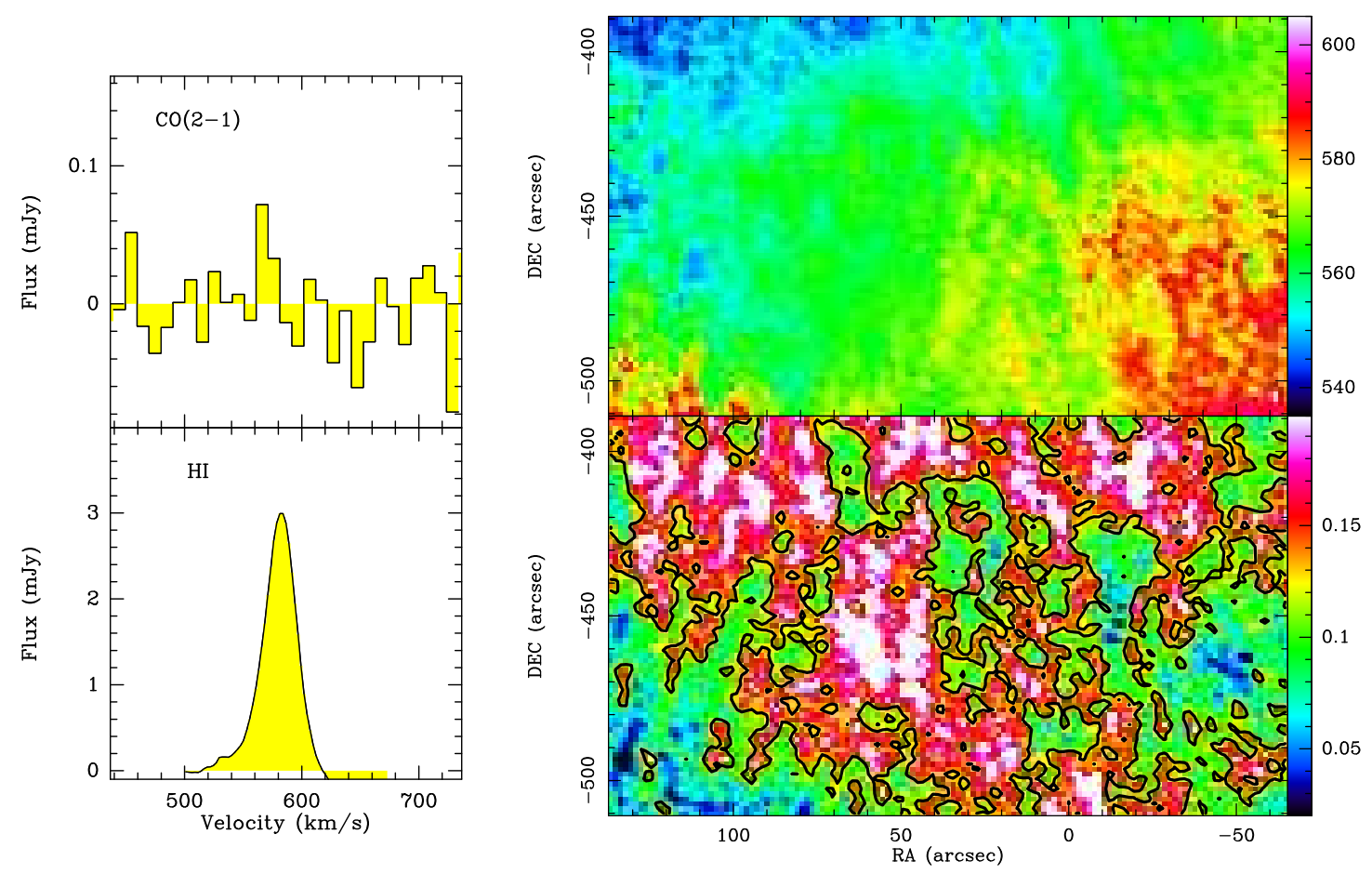

Fig. 5. Left panels: result of the stacking procedure for the $\mathrm{CO}(2-1)$ map (top) and the HI map (bottom). Only spectra with a significant HI detection have been considered (see text), and they have all been shifted to $580 \mathrm{~km} \mathrm{~s}^{-1}$ central velocity. Right panels: HI (THINGS) integrated intensity map $\left(\mathrm{Jy} \times \mathrm{km} \mathrm{s}^{-1}\right.$, bottom $)$ in the region of the mosaic observed by ALMA, and the corresponding average velocity $($ top $)$ in $\mathrm{km} \mathrm{s}^{-1}$. The spatial coordinates are in arcsec offset from the galaxy centre $\left(13^{\mathrm{h}} 37^{\mathrm{m}} 00.9^{\mathrm{s}},-29^{\circ} 51^{\prime} 56^{\prime \prime}\right)$. The HI beam is $15.2^{\prime \prime} \times 11.4^{\prime \prime}$ (VLA NA map from THINGS). The black contour corresponds to NHI $=10^{21} \mathrm{~cm}^{-2}$.

CO emission was detected in Cycle 2 ALMA maps. To analyse the impact of our observations in the context of star formation in outer XUV disks, we have investigated the behaviour in the Kennicutt-Schmidt diagram of the tentative $\mathrm{CO}$ detections, in plotting the equivalent molecular gas surface density, and in star formation surface density.

\subsection{Star formation diagram}

The SFR surface density can be determined from a combination of FUV and $24 \mu \mathrm{m}$ fluxes on timescales of $10-100 \mathrm{Myr}$, using the calibration from (Leroy et al. 2008):

$$
\begin{aligned}
\Sigma_{\mathrm{SFR}}\left(M_{\odot} \mathrm{yr}^{-1} \mathrm{kpc}^{-2}\right)= & 8.1 \times 10^{-2} F_{\mathrm{FUV}}\left(\mathrm{MJy} \mathrm{sr}^{-1}\right) \\
& +3.2 \times 10^{-3} F_{24 \mu \mathrm{m}}\left(\mathrm{MJy} \mathrm{sr}^{-1}\right)
\end{aligned}
$$

Within an extended HII region, the very recent SFR (on a 3-10 Myr timescale) can be determined from the $\mathrm{H} \alpha$ luminosity by (Kennicutt \& Evans 2012)

$\operatorname{SFR}\left(M_{\odot} \mathrm{yr}^{-1}\right)=5.37 \times 10^{-42} L(\mathrm{H} \alpha)\left(\mathrm{erg} \mathrm{s}^{-1}\right)$

The $\mathrm{H} \alpha$ luminosity was corrected for internal extinction, through $L(\mathrm{H} \alpha)_{\text {corr }}=L(\mathrm{H} \alpha)_{\mathrm{obs}}+0.020 L(24 \mu \mathrm{m})$, according to Kennicutt \& Evans (2012). Over an aperture of $200 \mathrm{pc}$ in size around the two strongest HII regions, we find an SFR of $3 \times 10^{-4}$ and $3.6 \times 10^{-4} M_{\odot} \mathrm{yr}^{-1}$. These values are comparable to that obtained from Eq. (1). The upper limits of $\mathrm{CO}$ emission are also averaged over the same area (200 pc in size) to compute the corresponding surface densities. Schruba et al. (2010) have studied the dependence of the molecular depletion time in M33 on the scale considered, and they conclude that $\sim 300 \mathrm{pc}$ is the limiting scale below which the SF law as a function of gas surface density is likely to break. We consider a very similar scale where the SF law should be relevant. We calculated the molecular hydrogen surface density as

$\Sigma_{\mathrm{H}_{2}}\left(M_{\odot} \mathrm{pc}^{-2}\right)=4.2 I_{1-0}\left(\mathrm{~K} \mathrm{~km} \mathrm{~s}^{-1}\right)$,

where $I_{1-0}$ is the $\mathrm{CO}(1-0)$ line intensity in $\mathrm{K} \mathrm{km} \mathrm{s}^{-1}$, and we assume that the $\mathrm{CO}(2-1)$-to- $\mathrm{CO}(1-0)$ intensity ratio is $R_{21}=$ $I_{21} / I_{10}=0.7$. With the spatial resolution of our observations, the flux scale is $0.018 \mathrm{Jy} \mathrm{K}^{-1}$. The standard CO-to- $\mathrm{H}_{2}$ conversion ratio of $X_{\mathrm{CO}}=2 \times 10^{20} \mathrm{~cm}^{-2} /\left(\mathrm{K} \mathrm{km} \mathrm{s}^{-1}\right)$ is adopted, and the number in Eq. (3) includes helium correction.

We plot the Kennicutt-Schmidt relation in Fig. 6, where we compare all the molecular data obtained in outer disks to date, with the large sample of nearby galaxies from Bigiel et al. (2008). The total gas surface density $\Sigma_{\mathrm{H}_{2}}+\Sigma_{\mathrm{HI}}$ is completely dominated by molecular gas above $9 M_{\odot} \mathrm{pc}^{2}$, and the SFR relation can then be considered as linear with the gas surface density.

For M83, the regions used for this plot are the most prominent star-forming regions in the $\mathrm{H} \alpha$ map. We smoothed the molecular gas surface density over the extent of the $\mathrm{H} \alpha$ region size, about $200 \mathrm{pc}$, which leads to lower upper limits. In these regions, the average SFR surface density is high enough that we expect to find only molecular gas. We then plotted only the upper limit on $\mathrm{H}_{2}$ surface density obtained by averaging over the $200 \mathrm{pc}$ region. For all other compared galaxies, only the molecular component was taken into account. It can be seen that the time to consume the molecular gas in the outer regions of M83 is less $\left(t_{\mathrm{dep}}<3 \times 10^{8} \mathrm{yr}\right)$ than the depletion time in nearby galaxies $\left(t_{\mathrm{dep}}=3 \times 10^{9} \mathrm{yr}\right)$. It is not relevant to represent in this diagram the average value obtained through the stacking over the whole region of $4 \mathrm{kpc} \times 2 \mathrm{kpc}$ since the surface densities are then diluted to a region much larger than the usual star-forming 


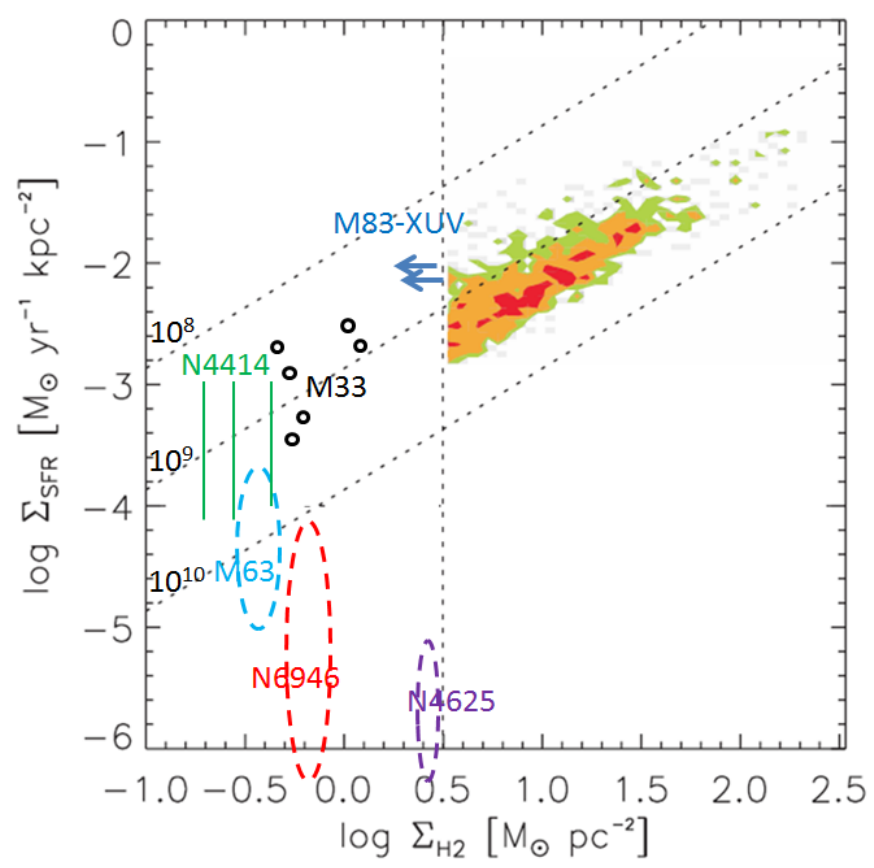

Fig. 6. Kennicutt-Schmidt diagram relating the SFR surface density to the molecular gas surface density, adapted from Verdugo et al. (2015) and Bigiel et al. (2008). Dashed ovals represent the data from the outer parts of XUV disk galaxies: NGC4625 and NGC6946 from Watson et al. (2016), and M63 (NGC 5055) from Dessauges-Zavadsky et al. (2014), taking only the molecular gas into account in all of them. The three green vertical bars are from NGC 4414 (Braine \& Herpin 2004), and the black circles for M33 (Gratier et al 2010). The dashed vertical line corresponds to $3 M_{\odot} \mathrm{pc}^{2}$, the sensitivity limit of the CO data in Bigiel et al. (2008). Dashed inclined lines correspond to depletion times of $10^{8}, 10^{9}$, and $10^{10}$ years to consume all the gas at the present SFR. The horizontal upper limits correspond to our $\mathrm{CO}(2-1)$ results on the M83 XUV disk of $\Sigma_{\mathrm{H}_{2}}$, in two of the main SFR regions, $200 \mathrm{pc}$ in size.

regions, used in this diagram (about $200-500 \mathrm{pc}$ in size). It is indeed on such scales that a star-forming region can be defined. At lower scales, the star formation tracers, such as $\mathrm{H} \alpha$ or FUV, are not expected to be correlated to the molecular clouds of their birth since newly born stars progressively drift away at a different velocity than the dissipative gas. For the other galaxies, the gas and SFR surface densities are also on such scales, for example M63 (Dessauges-Zavadsky et al. 2014) corresponding to the IRAM $30 \mathrm{~m}$ telescope beam of 0.5 and $1 \mathrm{kpc}$ in $\mathrm{CO}(2-1)$ and $\mathrm{CO}(1-0)$, respectively.

\subsection{Lack of $\mathrm{CO}$ emission in the outer disk of $\mathrm{M} 83$}

One main issue in trying to explain the absence of $\mathrm{CO}$ emission in galaxies is the metallicity $\mathrm{Z}$ of the gas. It is now well established that $\mathrm{CO}$ emission is frequently very faint or non-existent in low-metallicity galaxies, for example in gasrich irregular galaxies (Elmegreen et al. 1980; Tacconi \& Young 1987; Taylor et al. 1998). Observations and theory both indicate that the $\mathrm{CO}$ to $\mathrm{H}_{2}$ conversion factor, $X_{\mathrm{CO}}$, increases at low metallicity below $12+\log (\mathrm{O} / \mathrm{H})=8.2-8.4$ (Bolatto et al. 2013). The dependence of this relation is non-linear with $\mathrm{Z}$, and may be in $\mathrm{Z}^{-2}$ or steeper at low $\mathrm{Z}$ because of UV photo-dissociation in the absence of dust in addition to the underabundance of $\mathrm{CO}$.

However, in the M83 field observed with ALMA, there is only a slight abundance deficiency; the average metallicity of the $\mathrm{H}_{\mathrm{II}}$ regions is $12+\log (\mathrm{O} / \mathrm{H})=8.4$ (Bresolin et al. 2009), i.e. $1 / 2$ solar. With a slight change in $X_{\mathrm{CO}}$ for a higher value than standard, we should still be able to detect molecular clouds at more than $8 \sigma$. This means that the scatter in the $X_{\mathrm{CO}}-$ metallicity relation cannot explain the lack of $\mathrm{CO}$ emission.

The formation of the $\mathrm{CO}$ molecule occurs from $\mathrm{OH}$ via ionneutral reactions that form $\mathrm{HCO}+$, which after its dissociation forms $\mathrm{CO}$. Thus, the $\mathrm{CO}$ formation rate depends on the abundance of $\mathrm{OH}$, itself related to the rate of destruction by UV photons. The destruction of the CO molecule is mainly by photodissociation. In diffuse environments, the molecular clouds are more isolated, and less shielded. A schematic view of a molecular cloud includes a dense core, with $\mathrm{CO}$ emission, and a surface where $\mathrm{CO}$ is photodissociated, and emitting essentially in $\mathrm{C}+$ and $\mathrm{C}$ lines (see Fig. 8 in Bolatto et al. 2013). Because $\mathrm{H}_{2}$ and $\mathrm{CO}$ have similar dissociation processes in their line-transitions, $\mathrm{H}_{2}$ can partially shield the $\mathrm{CO}$ molecule, but with large $\mathrm{CO}$ column densities, the $\mathrm{CO}$ molecule is self-shielding. At low CO column densities, and in particular in the outer parts of molecular clouds, the $\mathrm{CO}$ molecule is absent, and the CO-to- $\mathrm{H}_{2}$ abundance ratio drops. All the carbon is found in $\mathrm{C}$ and $\mathrm{C}+$, and this gas is called CO-dark molecular gas.

Another possible scenario which could explain the lack of $\mathrm{CO}$ emission is that the photo-dissociated region is fragmented into smaller clouds. Each one does not have enough depth to have $\mathrm{CO}$ emission, and is dominated by $\mathrm{C}+$ emission. In outer disks, the small star-forming clouds will emit much less together for the same amount of gas than the larger clouds present within the optical disk. The same scenario has been invoked by Lamarche et al. (2017), who failed to detect CO emission towards the starbursting radio source $3 \mathrm{C} 368$ at $z=1.13$ using ALMA data. They discuss the possibility that they observed the far-infrared fine-structure oxygen lines in star-forming gas clouds before they had the chance to form an appreciable amount of $\mathrm{CO}$.

Watson et al. (2016) did not have a precise reason for not detecting $\mathrm{CO}$ in a young star-forming region of NGC4625, while Braine et al. (2007), Braine \& Herpin (2004), and Dessauges-Zavadsky et al. (2014) were able to detect weak CO emission in the outer parts of NGC 6946, NGC 4414, and M63, respectively. However, in M63 CO is detected only in 2 out of 12 XUV disk regions. Watson et al. (2016) conclude that, even if one explanation for the lack of $\mathrm{CO}$ emission in lower-mass galaxies can still be the low metallicity, the issue remains for the outskirts of massive galaxies. Deeper observations are needed to disentangle the various proposed scenarios.

The gas metallicity is only half solar in our mapped ALMA field, which could already reduce the size of the $\mathrm{CO}$ clouds with respect to $\mathrm{H}_{2}$ clouds. The lack of $\mathrm{CO}$ emission could also come from the excessive local FUV radiation field, which dissociates $\mathrm{CO}$ preferentially, and from the small size of the clouds in the outer regions of some galaxies. M83 shows particularly strong FUV emission in its outer regions with respect to others galaxies: the FUV flux ranges between 0.2 and $1.2 \times 10^{-2} \mathrm{MJy} \mathrm{sr}^{-1}$ over our region. The resolution of the FUV observations is $100 \mathrm{pc}$ at the distance of M83, so we cannot know exactly how much radiation is felt by each cloud. Although the FUV flux is much smaller than in the inner galaxy disk, clouds are smaller and less numerous in the outer parts of galaxies (here at half metallicity), which explains the more extended $\mathrm{CO}$ dissociation. This could be explored further through follow-up observations of the dust continuum in the Rayleigh-Jeans domain in the same region, another independent tracer of the molecular gas. 


\section{Conclusions}

We have reported on a mosaic of $\mathrm{CO}(2-1)$ observations obtained with ALMA in the M83 outer disk, rich in atomic gas and UV emission. M83 is the prototype of spiral galaxies with an extended XUV disk.

Our aims were to map $\mathrm{CO}$ emission in a small region $r_{\mathrm{gal}}=$ $11 \mathrm{kpc}$ from the galaxy centre. The result is a lack of $\mathrm{CO}$ emission, leading to the following conclusions:

1. An automatic search of $\mathrm{CO}$ emission in the data cube provides tentative detections of 14 clouds, but the $\mathrm{CO}(2-1)$ signal at $4-5 \sigma$ does not correspond spatially and spectrally to any other tracer. They are therefore considered false detections.

2. Conversely, we searched the CO map and extract the spectra at the peak of the star formation regions traced by $\mathrm{H} \alpha$. This did not provide any $\mathrm{CO}$ detection higher than $3 \sigma$.

3. We stacked all the $\mathrm{CO}$ pixels at the places where significant HI signal is found, shifting their velocity scale to a common central velocity expected from the HI signal. This gives a hint of a detection with a profile width of $\Delta V=14 \mathrm{~km} \mathrm{~s}^{-1}$. The corresponding $\mathrm{H}_{2}$ mass all over the $4 \times 2 \mathrm{kpc}$ area is only $2 \times 10^{6} M_{\odot}$. The $\mathrm{H}_{2}$-to-HI mass ratio over this region is $<3 \times 10^{-2}$

4. We display the $\mathrm{CO}$ upper limits towards the star-forming regions in the Kennicutt-Schmidt diagram, and the depletion time needed to consume the molecular gas is lower $\left(<3 \times 10^{8} \mathrm{yr}\right)$ than in normal galaxy disks $\left(3 \times 10^{9} \mathrm{yr}\right)$. We expected to find in some pixels of $17 \times 13 \mathrm{pc}$ GMC masses of $10^{6} M_{\odot}$, while the $3 \sigma$ upper limits are $\sim 10^{4} M_{\odot}$.

5. This lack of $\mathrm{CO}$ emission could be due partly to low metallicity since the gas abundance in this region of M83 is half solar. The average metallicity of the $\mathrm{H}_{\mathrm{II}}$ regions is $12+\log (\mathrm{O} / \mathrm{H})=$ 8.4 (Bresolin et al. 2009). Other causes of the lack in $\mathrm{CO}$ may be the strong UV field, and low global density of gas and dust; the gas is predominantly $\mathrm{H}_{2}$ but most carbon is not in $\mathrm{CO}$ molecules. In the photodissociation regions, the carbon is in $\mathrm{C}$ and $\mathrm{C}+$.

6. In the outer parts of galaxies, at low gas surface density, the clouds are likely to be smaller than in the disk, and less selfshielded. The CO column density in each cloud is then not sufficient to avoid dissociation, and the region is dominated by $\mathrm{C}+$ emission.

Acknowledgements. We are very grateful to the referee for the very useful comments which helped improve and clarify the paper. ICB would like to acknowledge the financial support from CAPES during this project. The ALMA staff in Chile and ARC-people at IRAM are gratefully acknowledged for their help in the data reduction. This paper makes use of the following ALMA data: ADS/JAO.ALMA\#2013.1.00861.S. ALMA is a partnership of ESO (representing its member states), NSF (USA), and NINS (Japan), together with NRC (Canada) and NSC and ASIAA (Taiwan), in cooperation with the
Republic of Chile. The Joint ALMA Observatory is operated by ESO, AUI/NRAO, and NAOJ. The National Radio Astronomy Observatory is a facility of the National Science Foundation operated under cooperative agreement by Associated Universities, Inc. M.R. would like to acknowledge support from CONICYT(CHILE) through FONDECYT grant No. 1140839 and partial support from project BASAL PFB-06.

\section{References}

Allen, R. J., Heaton, H. I., \& Kaufman, M. J. 2004, ApJ, 608, 314

Bigiel, F., Leroy, A., Walter, F., et al. 2008, AJ, 136, 2846

Bigiel, F., Leroy, A., Walter, F., et al. 2010, AJ, 140, 1194

Bigiel, F., Leroy, A. K., Walter, F., et al. 2011, ApJ, 730, L13

Boissier, S., Prantzos, N., Boselli, A., \& Gavazzi, G. 2003, MNRAS, 346, 1215

Boissier, S., Gil de Paz, A., Boselli, A., et al. 2007, ApJS, 173, 524

Bolatto, A. D., Wolfire, M., \& Leroy, A. K. 2013, ARA\&A, 51, 207

Braine, J., \& Combes, F. 1992, A\&A, 264, 433

Braine, J., \& Herpin, F. 2004, Nature, 432, 369

Braine, J., Ferguson, A. M. N., Bertoldi, F., \& Wilson, C. D. 2007, ApJ, 669 , L73

Bresolin, F., Ryan-Weber, E., Kennicutt, R. C., \& Goddard, Q. 2009, ApJ, 695, 580

Bresolin, F., Kudritzki, R.-P., Urbaneja, M. A., et al. 2016, ApJ, 830, 64

Crosthwaite, L. P., Turner, J. L., Buchholz, L., Ho, P. T. P., \& Martin, R. N. 2002, AJ, 123, 1892

Dessauges-Zavadsky, M., Verdugo, C., Combes, F., \& Pfenniger, D. 2014, A\&A, 566, A 147

Digel, S., de Geus, E., \& Thaddeus, P. 1994, ApJ, 422, 92

Dong, H., Calzetti, D., Regan, M., et al. 2008, AJ, 136, 479

Elmegreen, B. G., Morris, M., \& Elmegreen, D. M. 1980, ApJ, 240, 455

Gardan, E., Braine, J., Schuster, K. F., Brouillet, N., \& Sievers, A. 2007, A\&A 473, 91

Gil de Paz, A., Madore, B. F., Boissier, S., et al. 2005, ApJ, 627, L29

Gil de Paz, A., Boissier, S., Madore, B. F., et al. 2007a, ApJS, 173, 185

Gil de Paz, A., Madore, B. F., Boissier, S., et al. 2007b, ApJ, 661, 115

Gratier, P., Braine, J., Rodriguez-Fernandez, N. J., et al. 2010, A\&A, 522, A3

Kennicutt, Jr., R. C. 1998, ApJ, 498, 541

Kennicutt, R. C., \& Evans, N. J. 2012, ARA\&A, 50, 531

Koda, J., Yagi, M., Boissier, S., et al. 2012, ApJ, 749, 20

Lamarche, C., Stacey, G., Brisbin, D., et al. 2017, ApJ, 836, 123

Leroy, A. K., Walter, F., Brinks, E., et al. 2008, AJ, 136, 2782

Saintonge, A., Kauffmann, G., Wang, J., et al. 2011, MNRAS, 415, 61

Schmidt, M. 1959, ApJ, 129, 243

Schruba, A., Leroy, A. K., Walter, F., et al. 2011, AJ, 142, 37

Schruba, A., Leroy, A. K., Walter, F., Sandstrom, K., \& Rosolowsky, E. 2010, ApJ, 722, 1699

Smith, D. A., Allen, R. J., Bohlin, R. C., Nicholson, N., \& Stecher, T. P. 2000, ApJ, 538, 608

Sun, Y., Xu, Y., Yang, J., et al. 2015, ApJ, 798, L27

Sun, Y., Su, Y., Zhang, S.-B., et al. 2017, ApJS, 230, 17

Tacconi, L. J., \& Young, J. S. 1987, ApJ, 322, 681

Taylor, C. L., Kobulnicky, H. A., \& Skillman, E. D. 1998, AJ, 116, 2746

Thilker, D. A., Bianchi, L., Boissier, S., et al. 2005a, ApJ, 619, L79

Thilker, D. A., Bianchi, L., Boissier, S., et al. 2005b, ApJ, 619, L79

Verdugo, C., Combes, F., Dasyra, K., Salomé, P., \& Braine, J. 2015, A\&A, 582, A6

Vio, R., \& Andreani, P. 2016, A\&A, 589, A20

Walter, F., Brinks, E., de Blok, W. J. G., et al. 2008, AJ, 136, 2563

Watson, L. C., Martini, P., Lisenfeld, U., Böker, T., \& Schinnerer, E. 2016, MNRAS, 455, 1807

Zaritsky, D., \& Christlein, D. 2007, AJ, 134, 135 